\title{
Treatment of the microfilaraemia of asymptomatic brugian filariasis with single doses of ivermectin, diethylcarbamazine or albendazole, in various combinations
}

\author{
By R. K. SHENOY*, S. DALIA, A. JOHN \\ Filariasis Chemotherapy U nit, T.D. M edical College Hospital, Alappuzha 688 011, India
}

T. K. SUMA

Department of Medicine, T.D. Medical College Hospital, Alappuzha 688 011, India

AND V. KUMARASWAMI

Tuberculosis Research Centre, Chennai 600 031, India

Received 2 M arch 1999, Revised 17 M ay 1999,

Accepted 19 M ay 1999

Several new chemotherapeutic tools are now available for the control of lymphatic filariasis. Combinations of single doses of antifilarial drugs are generally superior to single drugs. The efficacy and safety of albendazole in combination with diethylcarbamazinc (DEC) or ivermectin, for the treatment of Brugia malayi infection, were investigated, for the first time, in an open, hospital-based study. Fifty-one asymptomatic microfilaraemics (with 108-4034 microfilariae/ml; median = 531) of both sexes and aged 14-70 years were randomly allocated to receive single-dose treatments of ivermectin $(200 \mu \mathrm{g} / \mathrm{kg}) \mathrm{with}$ diethylcarbamazine (DEC; $6 \mathrm{mg} / \mathrm{kg})$, ivermectin $(200 \mu \mathrm{g} / \mathrm{kg})$ with albendazole $(400 \mathrm{mg}), \mathrm{DEC}(6 \mathrm{mg} / \mathrm{kg})$ with albendazole (400 mg), or albendazole (400 mg) alone. Albendazole alone had no effect on the microfilarial levels at the 1-year follow-up but both groups given DEC had significantly lower microfilaraemias $(P<0.015$ and $P<0.02$ ) than that given ivermectin with albendazole. Overall, $47 \%-64 \%$ of those given DEC but only $14 \%$ of those given ivermectin with albendazole appeared to be amicrofilaraemic 1 year post-treatment. The adverse reactions seen in the study were mild, transient and qualitatively similar to those seen earlier with ivermectin and DEC. The combination of DEC and albendazole, both well tested drugs, offers a new option for countries such as India where there is no onchocerciasis or loiasis and where ivermectin may not be immediately available. The direct and indirect effects of albendazole on intestinal helminths would be additional benefits.

Lymphatic filariasis is regarded as a major health problem in many parts of the tropical world, with recent estimates indicating that nearly 119 million people are afflicted by the disease (Michael et al., 1996). After mood disorders, the disease is the most common cause of physical morbidity (WHO, 1995). Until recently, control of the disease was

\footnotetext{
* E-mail: rkshenoy@md3.vsnl.net.in; fax: + 91477 251353.
}

largely based on the use of a single drug, diethylcarbamazine (DEC), in various regimens (control of the mosquito vectors was largely unsuccessful, for a variety of reasons). However, several new developments in the chemotherapy of the disease have completely changed the outlook for its control. These developments followed the discovery that ivermectin, a semi-synthetic macrolide, was effective in clearing the parasitaemias of Wuchereria bancrofti (Kumaraswami et al., 
1988) and Brugia malayi infections (Shenoy et al., 1992). In a comparative study, single doses of iverrnectin were found to be as effective as the standard 12-day course of DEC (Ottesen et al., 1990). These results led to a reexamination of the older drug: DEC. Surprisingly, a single dose of DEC was found to be as effective as the standard course over 12 days (Andrade et al., 1995). Recent studies using ultrasound (Dreyer et al., 1995) and new antigen-detection assays (Weil et al., 1997) have also convincingly documented the macrofilaricidal potential of DEC, which had been suspected on clinical, parasitological and histopathological grounds (Ottesen, 1985). Furthermore, the utility of DEC-fortified salt as a tool for effective control has now been well documented (Gelband, 1994; Shenoy et al., 1998b).

The superiority of combinations of ivermectin and DEC over either drug used alone has been established, in the treatment of both bancroftian (Moulia-Pelat et al., 1995) and brugian infections (Shenoy et al., 1998a). More recently, it has been demonstrated that combinations of albendazole with either ivermectin or DEC can effectively clear the microfilaraemias of bancroftian infections (Addiss et al., 1997; Ismail et al., 1998). The aim of the present study was to see if albendazole, either alone or in combination with ivermectin or DEC, was as effective against brugian filariasis.

\section{SUBJECTS AND METHODS}

\section{Subjects}

Fifty-one individuals with Brugia malayi microfilaraemias participated in the present, hospital-based study. Since all the three drugs tested-albendazole, ivermectin and DECare already being used in communities, it was decided to include (non-pregnant, non-lactating) females and children in the study, so that these groups could also benefit from the results. Each of the subjects (adults) or their parents (children) gave informed consent. None of the subjects had any clinical or labo- ratory abnormalities other than their microfilaraemia. The intensities of the microfilaraemias on enrollment ranged from 108-4064 microfilariae $(\mathrm{mff}) / \mathrm{ml}$ $($ median $=531 \mathrm{mff} / \mathrm{ml})$. The subjects were recruited after night-blood screening at special Microfilaria Detection Camps organized by the Filariasis Chemotherapy Unit (FCU) of the T.D. Medical College Hospital, Alappuzha, India, and were residents of an area known to be endemic for brugian filariasis.

\section{Clinical and Laboratory Examinations}

Each subject was hospitalized for 7 days in the ward of the FCU, to permit extensive clinical and laboratory investigations, accurate drug administration and monitoring of any adverse reactions (Shenoy et al., 1992, 1993, 1998a, b). On admission, each subject was given a complete physical examination, haematological screening (haematocrit, complete blood counts, and total and differential white-bloodcell counts) and biochemical tests (bilirubin, aspartate aminotransferase, alanine aminotransferase, alkaline phosphatase and creatinine levels) and subjected to chest radiography and electrocardiography. Vital signs were monitored every $4 \mathrm{~h}$ for the first $48 \mathrm{~h}$ post-treatment and then each morning during the hospitalization. Signs and symptoms were graded 0 (none), 1 (mild) or 2 (severe) at each follow-up and the grades recorded during the hospitalization were summed to give a total score for each subject (Shenoy et al., 1992, 1993, 1998a). All the subjects were required to return to the hospital at frequent intervals as part of the followup programme. At each visit they were questioned about signs and symptoms and clinically evaluated. All the biochemical tests given on admission were repeated 2 weeks post-treatment to evaluate the safety of the trial drugs.

\section{Study Design}

Initially, the 51 subjects were randomly allocated (using block randomization) to one of four single-dose treatments: ivermectin $(200 \mu \mathrm{g} / \mathrm{kg})$ with DEC (6 $\mathrm{mg} / \mathrm{kg})$; ivermectin 
$(200 \mu \mathrm{g} / \mathrm{kg})$ with albendazole $(400 \mathrm{mg})$; DEC $(6 \mathrm{mg} / \mathrm{kg})$ with albendazole $(400 \mathrm{mg})$; or albendazole $(400 \mathrm{mg}$ ) alone. After the study had commenced, however, the albendazoleonly treatment arm was dropped after only three patients had been allocated to it, because of a change in the study design. However, the three patients continued to be followed up, in a manner similar to the others in the study.

The drugs were administered at 08.00 hours on the third hospital day (day 3), on an empty stomach. They were given as tablets, each containing 50 or $100 \mathrm{mg}$ DEC, $6 \mathrm{mg}$ ivermectin or $400 \mathrm{mg}$ albendazole. The mean total doses given per subject were $308 \mathrm{mg}$ DEC $(5.99 \mathrm{mg} / \mathrm{kg}), 10.4 \mathrm{mg}$ ivermectin $(201.55 \mu \mathrm{g} / \mathrm{kg}$ ) and/or $400 \mathrm{mg}$ albendazole. Alcoholic beverages were not permitted on days 2-10. There were no other dietary restrictions. Severe reactions were treated with paracetamol but no other medication was administered for 7 days post-treatment. None of the subjects had previously received antifilarials and no such drugs or antiparasitic drugs were allowed for 1 year post-treatment.

\section{Efficacy Measurements}

Microfilarial densities ( $\mathrm{mff} / \mathrm{ml}$ blood) were used as indices of efficacy and were determined by diluting $1 \mathrm{ml}$ heparinized venous blood with $3 \mathrm{ml}$ saline and filtering $1 \mathrm{ml}$ of the mixture through a $3-\mu \mathrm{m}$-pore membrane filter (Nuclepore). The microfilariae trapped on the filter were then counted. The pre-treatment microfilarial density for each subject was estimated as the mean value obtained using three blood samples collected in the week prior to drug administration. Microfilarial densities were subsequently determined 1, 2, 5, 7, 14, 30, 90, 180, 270 and 360 days post-treatment.

\section{Statistical Methods}

All microfilarial counts were logarithmically transformed before comparison by Student's t-tests, and expressed as percentages of the corresponding pre-treatment values when appropriate.

\section{RESULTS}

The 51 subjects enrolled were aged 14-70 years. The data for six subjects were excluded from the final analysis because job constraints made it impossible for the subjects to adhere to the follow-up schedule (one patient given ivermectin with DEC, one given ivermectin with albendazaole, and one given DEC with albendazole), because the subjects had received inadvertent treatment with DEC elsewhere during the study period (one subject given ivermectin with albendazaole, and one given albendazole alone), or because of emigration from the study area (one subject given ivermectin with DEC). The data on the remaining 45 patients who successfully completed a year of follow-up (i.e. 14 given ivermectin with DEC, 14 given ivermectin with albendazole, 15 given DEC with albendazole, and just two given albendazole alone) were included in the efficacy analysis. [At the end of the present study, 1 year posttreatment, each of these 45 subjects was given albendazole with DEC (those previously given albendazole alone) or identical treatment to that given previously. Each will be followed-up for another year.]

The age and sex distributions and the pretreatment microfilarial densities in the four treatment groups are shown in the Table. The mean pre-treatment density in the subjects belonging to the ivermectin + DEC group was significantly higher than the mean densities for the other three groups $(\mathrm{P}<0.01$ for each), which were similar.

\section{Microfilarial Clearance}

The pattern of microfilarial clearance in each of the treatment groups is illustrated in the Figure. The combination of ivermectin and DEC achieved a mean clearance of $99.56 \%$ by $12 \mathrm{~h}$ post-treatment, reaching $100 \%$ by that time in five subjects. Ivermectin with albendazole gave a mean clearance of only $78.87 \%$ by $12 \mathrm{~h}$ post-treatment and all the subjects given this combination remained microfilaraemic at that time. In the DEC + albendazole group the mean clearance $12 \mathrm{~h}$ post-treatment was 
TABLE

The ages, sexes and treatments of the 51 subjects and the levels of their microfilaraemias pre-treatment

\begin{tabular}{|c|c|c|c|c|c|}
\hline \multirow[b]{2}{*}{ Treatment } & \multicolumn{3}{|c|}{ No. of subjects } & \multirow{2}{*}{$\begin{array}{c}\text { Age range } \\
\text { (years) }\end{array}$} & \multirow{2}{*}{$\begin{array}{c}\text { Geometric mean } \\
\text { microfilaraemias and (ranges) } \\
\text { (microfilariae/ml) }\end{array}$} \\
\hline & Males & Females & All & & \\
\hline Ivermectin + diethylcarbamazine & 13 & 3 & 16 & $16-55$ & $912(236-4064)$ \\
\hline Ivermectin + albendazole & 14 & 2 & 16 & $14-70$ & $482(108-1981)$ \\
\hline Diethylearbamazine + albendazole & 14 & 2 & 16 & $15-65$ & $374(145-978)$ \\
\hline Albendazole & 3 & 0 & 3 & $46-48$ & $306(181-747)$ \\
\hline
\end{tabular}

$96.16 \%$ but only one subject was amicrofilaraemic. There was practically no clearance of microfilariae in the three patients given albendazole alone.

Microfilaraemias in those given ivermectin + DEC dropped rapidly and remained low throughout the follow-up year (with densities only $0.3 \%-4.2 \%$ of those seen pre-treatment). The decline in microfilarial densities in those given ivermectin with albendazole was more gradual, reaching a minimum $(2.2 \%$ of the pre-treatment value) 30 days posttreatment; thereafter the levels increased steadily until the end of the follow-up year. The minimum mean microfilarial density seen in those given DEC + albendazole occurred $12 \mathrm{~h}$ post-treatment, the mean densities then slowly rose until 7 days post-treatment (to $14.6 \%$ of the pre-treatment level) before gradually falling back down. In the small group that received albendazole alone, the mean values fluctuated widely but remained high $(82.6 \%-148 \%$ of the mean pre-treatment level).

At the end of the first year, the mean microfilarial levels in the two groups given DEC were low $(0.33 \%$ of the pre-treatment value for ivermectin + DEC and $1.51 \%$ for DEC + albendazole). Although one was not statistically different from the other $(\mathrm{P}>0.5)$, both were significantly lower than the level recorded at the same time in those given ivermectin + albendazole $(\mathrm{P}<0.020$ for each).

One year post-treatment, nine $(64 \%)$ of the 14 patients given ivermectin + DEC, seven
(47\%) of the 15 given DEC + albendazole but only two $(14 \%)$ of the 14 given ivermectin + albendazole and neither of those who received albendazole alone were amicrofilaraemic.

\section{Adverse Reactions}

The data for all 51 subjects who were initially enrolled on the study were included in the analysis of adverse reactions since all were available for investigation until 1 month posttreatment, by which time all side-effects had disappeared. Adverse effects first appeared about $12 \mathrm{~h}$ post-treatment but generally cleared within $48 \mathrm{~h}$. The most frequently reported reactions were fever, headache, myalgia, chills and lethargy. Most did not require any treatment other than paracetamol. Adverse reactions were reported by all 16 subjects given ivermectin + DEC, $12(75 \%)$ of the 16 given ivermectin + albendazole, $15(94 \%)$ of those given DEC + albendazole and two $(67 \%)$ of the three subjects who received albendazole alone. Those given albendazole alone only complained of mild myalgia. The mean (SD.) adverse-reaction scores for the groups were as follows: 8.6 (5.7) for ivermectin + DEC; 3.9 (5.1) for ivermectin + albendazole; 3.8 (2.1) for DEC + albendazole; and 3.3 (1.7) for albendazole alone. The mean score for those given ivermectin + DEC was significantly higher than those for the subjects who received ivermectin + albendazolc $\quad(\mathrm{P}<0.021) \quad$ or DEC + albendazole $(\mathrm{P}<0.003)$. 


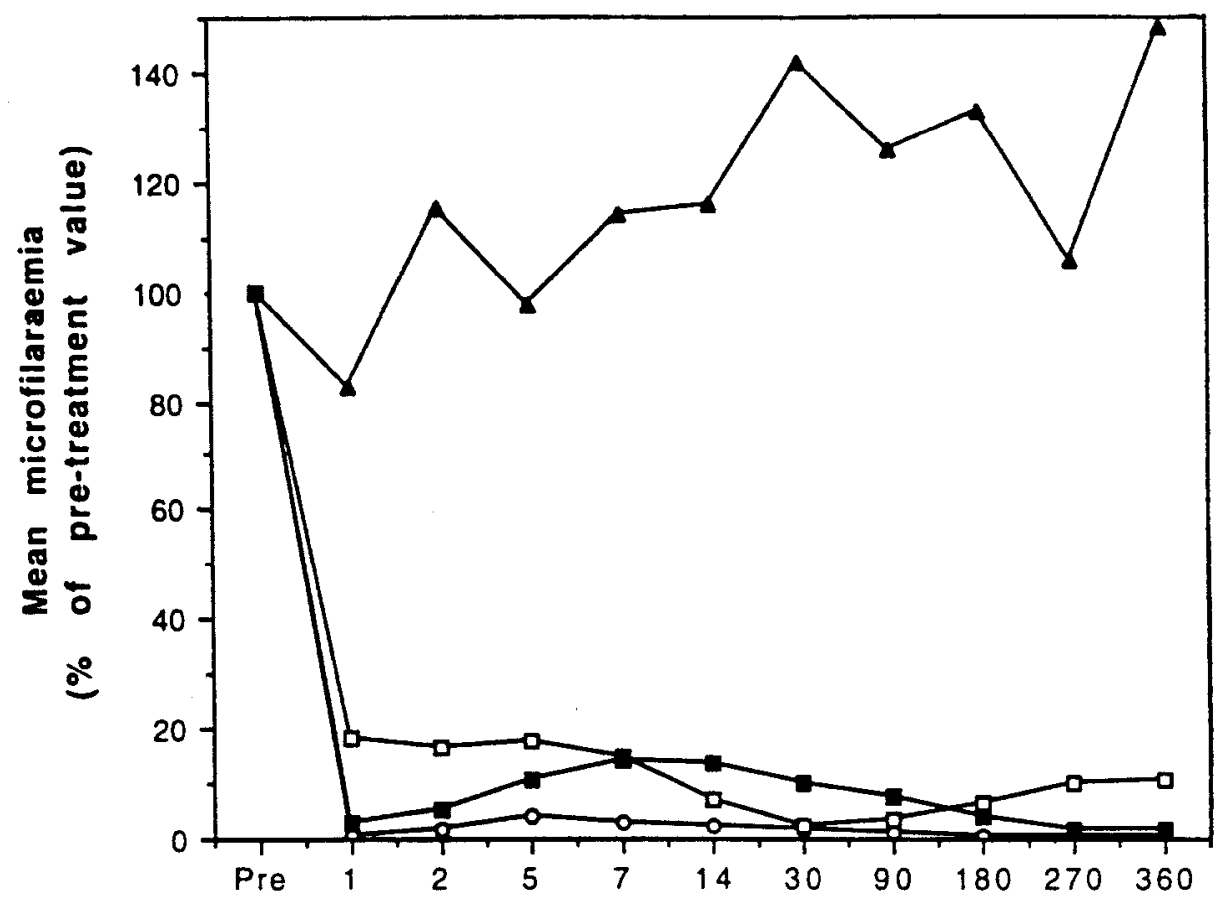

Days post-treatment

Fig. Microfilarial clearance in those treated with ivcrmcctin + diethylcarbamazine (0), ivermectin + albendazolc $(\mathrm{O})$, albendazole + diethylearbamazinc $(\mathrm{m})$ or albendazolc alone (A).

\section{DISCUSSION}

The chemotherapeutic tools available for the control of lymphatic filariasis have rapidly increased in number during the last decade. Effective parasite control can now be achieved using single annual doses of DEC or ivermectin, either alone or in combination (Ottescn and Ramachandran, 1995). DECfortified salt is another option for the control of bancroftian (Gelband, 1994) and brugian infections (Shenoy et al., 1998b). New diagnostic tools, such as DNA probes (WHO, 1994) and tests to detect daytime-circulating antigen (Weil et al., 1997), which are potentially useful in monitoring the success of control programmes, have also been developed. These advances have been responsible for the identification of lymphatic filariasis as one of only six potentially eradicable diseases (Anon.,
1993) and the recent passage of a World Health Assembly resolution to eliminate this disease as a public-health problem (Ottesen, 1998).

The recent observations that albendazole, in combination with either ivermectin (Addiss et al., 1997; Ismail et al., 1998) or DEC (Ismail et al., 1998), can suppress bancroftian microfilaracmia for prolonged periods has opened up the possibility of using this drug in large-scale control programmes. Since albendazolc is a broad-spectrum anthclmintic with proven efficacy against a range of intestinal nematodes, its use would have the additional benefit of clearing these parasites from the treated individuals. Use of albendazole has particular appeal to those involved in school health programmes aimed at control of geohelminth infections, since it would help the children treated to get rid of their filarial 
parasites early and thus prevent morbidity later (Addiss et al., 1997).

The present study appears to be the first to investigate the efficacy and safety of albendazole, either alone or in combination with other antifilarial drugs, in the control of brugian filariasis. There are known to be significant differences between brugian and bancroftian infections in terms of the efficacy of, and tolerance to their treatment with antifilarial drugs, whether these are used singly (Shenoy et al., 1992), in combinations (Shenoy et al., 1998a) or as fortified salt (Shenoy et al., 1998b). The present results again highlight the differences in responses of these two infections and also the need for developing appropriate control strategies for brugian filariasis.

The most rapid and best sustained suppression of microfilaraemia was seen in the group treated with ivermectin and DEC and was similar to that seen in an earlier study in which the same combination was used (Shcnoy et al., 1998a). However, at the end of 1 year there was no difference between the microfilarial clearance in this group and that in the subjects who received DEC + albendazole $(\mathrm{P}>0.5)$. The pattern of clearance caused by the two DEC-containing regimens was similar: a rapid decline in counts in the first $12 \mathrm{~h}$ post-treatment followed by a marginal rise (more pronounced in the $\mathrm{DEC}+$ albendazole group) between 2 to 14 days post-treatment. This effect was probably DEC-driven, reflecting an initial microfilaricidal action followed by a macrofilaricidal one (Shenoy et al., 1998a). The pattern of clearance produced by ivermectin + albendazole (a steady decline in microfilarial densities, for 30 days, followed by a slow but steady rise until the end of the 1-year follow-up), was similar to that observed when patients with brugian infections were treated with ivermectin alone (Shenoy et al., 1992). Albendazole, when given alone as a single, 400-mg dose, clearly had no effect on microfilarial levels. It therefore appears that it is the other drug (DEC or ivermectin) that plays the major role when the microfilaraemias of brugian infections are cleared using drug combinations including albendazole. More importantly, combinations of DEC with either ivermectin or albendazole are superior to a combination of ivermectin and albendazole in decreasing the intensity and prevalence of microfilaraemia 1 year post-treatment.

The adverse reactions seen in the present study were qualitatively similar to those seen when ivermectin and DEC arc given either singly or in combination (Shenoy et al., 1992, 1998a). The mean adverse-reaction score was highest in the group that received ivermectin + DEC. Unfortunately, however, because of the randomness of the allocation process, this group was also the one that had significantly higher microfilaraemias pretreatment than the other treatment. groups. Despite this limitation, the relatively high adverse-reaction scores of those given ivermectin + DEC probably reflect the systemic response to the rapid clearance of microfilaraemia caused by this drug combination.

No unusual side-effects, such as postural hypotension or the occurrence of the 'string sign', were seen during the present study, although Shenoy et al. (1998a) encountered these with a combination of ivermectin and DEC. No adverse reactions indicative of a macrofilaricidal effect (such as the development of nodules, lymphadenopathy, or a 'scrotal syndrome') were produced by any of the present treatments.

The present results highlight several issues relating to the mode of action of albendazole when combined with DEC or ivermectin for the treatment of microfilaraemias. Although albendazole undoubtedly has useful activity against intestinal helminths (Addiss et al., 1997), its role as an antifilarial in combination regimens with ivermectin or DEC needs to be examined carefully; it is unclear how it potentiates the antifilarial action of the other two drugs. Although albendazole used alone in repeated, high doses has been shown to be macrofilaricidal in bancroftian filariasis (Jayakody et al., 1993), the drug had no apparent effect on B. malayi in the present study. In contrast, DEC has been shown to have marked macrofilaricidal and microfilaricidal properties (Ottesen, 1985; Dreyer et al., 1995; Weil et al., 1997). Although ivermectin rapidly 
and effectively clears microfilaraemias and prevents their re-appearance for prolonged periods, there is no evidence to indicate that ivermectin has any macrofilaricidal activity. Since DEC + albendazole was at least as effective at clearing microfilaraemias as ivermectin + DEC in the present study, albendazole presumably enhances the activity of DEC. It would be interesting to observe the long-term effects (i.e. those occurring beyond 1 year) of DEC + albendazole, since the pattern of microfilarial clearance achieved with this combination in the present study (see Fig.) indicates that it may be even more effective than ivermectin + albendazole.

It is unfortunate that there are no markers to detect macrofilaricidal activity in antifilarial drugs when they are used against brugian infections. It is now possible to determine the adulticidal effect of drugs used, singly or in combination, in bancroftian filariasis, using either ultrasound (Dreyer et al., 1995) or antigen levels (Weil et al., 1997; Ismail et al., 1998). However, adult B. malayi do not appear to be detectable by ultrasound, and no corresponding antigen-detection tests are currently available for brugian infections (unpubl. obs.). Conclusions regarding the macrofilaricidal effects of any drug used against brugian filariasis must therefore be based on indirect evidence, such as long-term suppression of microfilaraemia.

The present observations have two important implications for programmes to control lymphatic filariasis in areas where brugian infections occur. Firstly, the combination of DEC and albendazole should be an efficient tool for the control of the microfilaraemias of brugian infections, being at least as effective as ivermectin with DEC. It will also be a particularly attractive tool in national programmes that target schoolchildren and young adults, because of the additional benefits of using albendazole. In addition to its well documented effects on intestinal helminths and the anaemia that. they produce (WHO, 1996), albendazole offers other benefits 'beyond filariasis'. These include gains in height and weight of treated children (Beach et al., 1998), improved performance at school (Nokes et al., 1992), and the increased productivity of the adults in treated populations (Stephenson et al., 1993). Secondly, and perhaps more importantly, the findings offer a new option for countries, such as India, which may not have ready access to ivermectin in the near future. In these countries, where DEC can safely be used since there is no onchocerciasis or loiasis, use of DEC + albendazole should provide all the advantages of a 'combination therapy' with the added advantage of using two drugs that are already used extensively and that have an excellent safety record. However, further, large-scale clinical trials will be needed before this combination can be used under programme conditions.

ACKNOWLEDGEMENTS. We would like to thank Dr E. A. Ottesen, Acting Team Co-ordinator (CEE/FIL) and Dr C. P. Ramachandran, previously Manager, Filariasis Field Trial Task Force, TDR/WHO, for their encouragement and valuable advice. We gratefully acknowledge the sociological help received from P. S. Ajithakumari and P. S. Sini, the technical assistance of S. Bindhu Thomas and the secretarial help of S. Sindhu. Our thanks are also due to the Principal and Superintendent of T.D. Medical College and Hospital for their support and encouragement. This study was supported by the UNDP/World Bank/WHO Special Programme for Research and Training in Tropical Diseases.

\section{REFERENCES}

Addiss, D. G., Beach, M. J., Streit, T. G., Lutwic, S., LeConte, F. H., Lafontant, J. G., Hightower, A. W. \& LAMmiE, P. J. (1997). Randomised placebo-controlled comparison of ivermectin and albendazole alone and in combination for Wuchereria bancrofti microfilaremia in Haitian children. Lancet, ii, 480-484. 
Andrade, L. A., Medeiros, Z., Pires, M. L., Pimentel, A., Rocha, A., Figuerado-Silva, J., Coutinho, A. \& Dreyer, G. (1995). Comparative efficacy of three different diethylcarbamazine regimens in lymphatic filariasis. Transactions of the Royal Society of Tropical Medicine and Hygiene, 89, 319-321.

AnON. (1993). Recommendations of the International Task Force for Disease Eradication. Morbidity and Mortality Weekly Report, 42, 1-38.

Beach, M. J., Streit, T. G., Addiss, D. G., Prospere R., Roberts, J. M. \& Lammie, P. J. (1998). Assessment of combined ivermectin and albendazole for the treatment of intestinal helminth and Wuchereria bancrofti infections in Haitian school children. American Journal of Tropical Medicine and Hygiene, 60, 479-486.

Dreyer, G., Amaral, F., Noroes, J., Medeiros, Z. \& Addiss, D. G. (1995). A new tool to assess invivo the adulticidal efficacy of antifilarial drugs for bancroftian filariasis. Transactions of the Royal Society of Tropical Medicine and Hygiene, 89, 225-226.

Gelband, H. (1994). Diethylcarbamazine salt in the control of lymphatic filariasis. American Journal of Tropical Medicine and Hygiene, 50, 655-662.

Ismail, M. M., Jayakody, R. L., Weil, G. J., Nirmalan, N., Jayasinghe, K. S. A., Abeyewickrema, W., Rezvi Sheriff, M. H., Rajaratnam, H. N., Amarasekara, N., De Silva, D. C. L., Michalski, M. L. \& DissanAike, A. L. (1998). Efficacy of single dose combinations of albendazole, ivermectin and diethylcarbamazine for the treatment of bancroftian filariasis. Transactions of the Royal Society of Tropical Medicine and Hygiene, 92, 94-97.

Jayakody, R. L., DE Silva, C. S. S. \& Werasinghe, W. M. T. (1993). Treatment of bancroftian filariasis with albendazole: evaluation of efficacy and adverse reactions. Tropical Biomedicine, 10, 19-24.

Kumaraswami, V., Ottesen, E. A., Vijayasekharan, V., Umadevi, S., Swaminathan, M., Aziz, M. A., SARMA, G. R., PrabhakAR, R. \& Tripathy, S. P. (1988). Ivermectin for the treatment of Wuchereria bancrofii filariasis: efficacy and adverse reactions. Journal of the American Medical Association, 259, 3150-3153.

Michael E., Bundy, D. A. P. \& Grenfell, B. T. (1996). Reassessing the global prevalence and distribution of lymphatic filariasis. Parasitology, 112, 409-428.

Moulia-Pelat, J. P., Glaziou, P., Weil, G. J., Nguyen, L. N., Gaxotte, P. \& Nicolas, L. (1995). Combination of ivermectin plus diethylcarbamazine, a new effective tool for control of lymphatic filariasis. Tropical Medicine and Parasitology, 46, 9-12.

Nokes, C., Grantham-McGregor, S. M., Sawyer, A. W., Cooper, E. S., Robinson, B. A. \& Bundy, D. A. P. (1992). Moderate to heavy infections of Trichuris trichura affect cognitive function in Jamaican school children. Parasitology, 104, 539-547.

OtTESEn, E. A. (1985). Efficacy of diethyicarbamazine in eradicating infection with lymphatic-dwelling filariae in humans. Reviews of Infectious Diseases, 7, 341-355.

Ottesen, E. A. (1998). Towards elimination of lymphatic filariasis. In Infectious Diseases and Public Health, eds Angelico, M. \& Rocchi, G. pp. 58-64. Tel Aviv: Balaban.

Ottesen, E. A. \& Ramachandran, C. P. (1995). Lymphatic filariasis infection and disease: control strategies. Parasitology Today, 11, 129-131.

Ottesen, E. A., Vijayasekharan V., Kumaraswami, V., Perumal Pillai, S. V., Sadanandam, A., Frederick, S., Prabhakar, R. \& Tripathy, S. P. (1990). A controlled trial of ivermectin and diethylcarbamazine in lymphatic filariasis. New England Journal of Medicine, 322, 1113-1117.

Shenoy, R. K., Kumaraswami, V., Rajan, K., Thankom, S. \& Jalajakumari (1992). Ivermectin for the treatment of periodic Malayan filariasis: a study of efficacy and side effects following a single oral dose and retreatment at six months. Annals of Tropical Medicine and Parasitology, 86, 271-278.

Shenoy, R. K., Kumaraswami, V., Rajan, K., Thankom, S. \& Jalajakumari (1993). A comparative study of the efficacy and tolerability of single and split doses of ivermectin and diethylcarbamazine in periodic brugian filariasis. Annals of Tropical Medicine and Parasitology, 87, 459-467.

Shenoy, R. K., George, L. M., John, A., Suma, T. K. \& Kumaraswami, V. (1998a). Treatment of microfilaraemia in asymptomatic brugian filariasis: the efficacy and safety of the combination of single doses of ivermectin and diethylcarbamazine. Annals of Tropical Medicine and Parasitology, 92, 579-585.

Shenoy, R. K., Varghese, J., Kuttikal, V. V. \& Kumaraswami, V. (19986). The efficacy, tolerability and safety of diethylcarbamazine-fortified salt in the treatment of the microfilaraemias of brugian filariasis: an open, hospital-based study. Annals of Tropical Medicine and Parasitology, 92, 285-293. 
Stephenson, L. S., Latham, M. C., Adams, E. J., Kinoti, S. N. \& Pertet, A. (1993). Physical fitness, growth and appetite of Kenyan school boys with hookworm, Trichuris trichura and Ascaris lumbricoides infections are improved four months after a single dose of albendazole. Journal of Nutrition, 123, 1036-1046.

Weil, C, Lammie, P. J. \& Weiss, N. (1997). The ICT filariasis test: a rapid format antigen test for diagnosis of bancroftian filariasis. Parasitology Today, 13, 401-404.

World Health Organization (1994). Lymphatic Filariasis: Infection and Disease: Control Strategies. Document TDR/CTD/FIL/Penang/94.1/1994. Geneva: WHO.

World Health ORganization (1995). The World Health Report: Bridging the Gap. Geneva: WHO.

World Health ORganization (1996). Report of the WHO Informal Consultation on Hookworm Infection and Anemia in Girls and Women. Document WHO/CTD/SIP96.1. Geneva: WHO. 